\title{
THE IMPACT OF WAITING TIME ON PEDESTRIAN VIOLATIONS AT SIGNALIZED INTERSECTIONS
}

\author{
Shuyan Chen, Jiping Xing and Yang Cao \\ School of Transportation, Southeast Univ., Nanjing, Jiangsu Province, China.
}

\begin{abstract}
The phenomenon that pedestrian violates traffic signals at intersections and crosses with danger is universal. This study aims to find out the influence of the waiting time of pedestrians at an intersection and the three main factors of pedestrian violations. In this study, 13 intersections and 1075 violations sample were collected in Suzhou China, which had different maximum waiting times. The validation would use the complementary video data. There are several factors, which have great influence on the proportion of pedestrian violations, such as the age, sex, arrive time, red phase, conflicting traffic volume and the length of volume crosswalks. Additionally, The significance of pedestrian maximum waiting time was highlighted in the end. For the purpose of reducing dangerous pedestrian behaviors at signalized intersections, minimizing waiting times is supposed to be one proper way.
\end{abstract}

\section{KEYWORDS}

Pedestrians ;Violations; Signalized intersections; Crossing Behaviour;

\section{INTRODUCTION}

In China, the crucial proportion of total traffic related injuries is related to pedestrian injuries. Currently, pedestrians' safety in urban areas has gained increasing concern. Meanwhile, the most vulnerable users for pedestrians are signalized intersections that are in urban areas. Besides, approximately sixty percentages of the situations pedestrians are wounded happened at intersections in cities. As the result of the fact that intersections are the most vulnerable places where pedestrians are exposed to motorized traffic, the intersection becomes the core roadway factor which is related to the high concentration of vehicle pedestrian crashes. It is widely known that intersections are complexity traffic environments. Under the impacts of one or multiple contributing elements, such as road design, human behaviour, and built an environment, pedestrians can be injured.

It has been highlighted in a large amount of recent studies that the factors, such as built environment and road, play an important role on pedestrian safety. Meanwhile, the significance of human factors is also demonstrated in some other studies. In addition, the elements associated with pedestrians' risky behaviors, such as red-light violations, have already been investigated in a few past studies.

In spite of the increasing studies on this subject, only very limited empirical researches investigate deeply into the time of pedestrians' arrival at intersections (the moment during pedestrians reach to signal intersections) and impacts of the waiting time (red phase). Meanwhile, 
depending on moments of the crossing with respects to the pedestrians' signal, violations have not been classified and modelled by previous studies, which only have analysed a very small sample of intersections. In addition, past studies have rarely explored the implantation of video data.

In this paper, factors such as time of arrival, time of departure, and maximum waiting time, as well as the analysis of pedestrian violations at signalized intersections, which have been rarely studied, are presented. Simultaneously, based on arrival time during the cycle, previous studies are added in this study by examining wait times. Moreover, one of the largest consideration in this type of study is the sample of studied intersections. The study will model violation probability by utilizing the discrete choice modelling approach. In addition, the study will collect the video data, which will be processed for validation, for two intersections.

In the next section, the background of this work will be presented, followed by an interpretation of the recommended methodology. Then come the presentation and discussion of the experimental results. The final chapter is the conclusion.

\section{PROBLEM DESCRIPTION}

Factors associated with pedestrian crossing behavior have been widely studied. These factors are related to the individual characteristics, environment, and other pedestrians' and road users' behavior, and may have combined effects. Studies have determined that men have a tendency to commit more violations than women (Moyano Diaz, 2002; Rosenbloom, 2009; Tiwari et al., 2007; Yagil, 2000) and young adults tend to violate a traffic light more often than other age groups. As people age, they are less likely to take risks while crossing the road, due in part to their decreasing mobility (Guo et al., 2011; Zhuang \& Wu, 2011). The people surrounding a pedestrian will also affect her or his crossing behavior. The larger the group of pedestrians, either traveling together or waiting at an intersection, the less likely a pedestrian is to violate the traffic control (Rosenbloom, 2009; Zhuang \& Wu, 2011). Teenagers, however, are more likely to violate traffic control in a larger group: Rosenbloom (2009) suggests that teenagers traveling with their peers tend to take bigger risks for reasons such as social acceptance. The trip purpose is also likely to impact the decision to commit violations. People traveling to work or school are more likely to commit violations than people traveling leisurely (Guo et al., 2011).

A pedestrian's decision to violate traffic signalization will also be affected by the characteristics of the intersection. Factors such as the presence of a pedestrian signal (Cambon de Lavalette et al., 2009; Markowitz, Sciortino, Fleck, \&Yee, 2006) and of countdown displays (Lipovac et al.,in press) have been proven to reduce the proportion of violations. Research has also been undertaken on the waiting duration. Van Houten et al. (2007) examined the relationship between pedestrian waiting time and violations at two signalized mid-block crosswalks. Minimum vehicle green time, i.e. pedestrian minimum waiting time, was manipulated from $30 \mathrm{~s}$ to 1 and $2 \mathrm{~min}$, and it was found that violation rates increased when the waiting time increased. Similar conclusions were drawn from a study at seven intersections in India (Tiwari et al.,2007). Recent studies have also been done in China. Based on observations at five intersections, Wang et al. (2011) found that people who had violated the traffic signal had a slightly smaller waiting time threshold on average; in a more recent study and using survey data, Ren et al. (2011) studied crossing behaviors at signalized intersections in three cities. They found that the largest proportion of surveyed individuals $(30.25 \%)$ indicated that they violated traffic rules to save time and for convenience. It is only in cases where the legal crossing must be made in two phases, that pedestrians would have much smaller waiting times if they committed a violation (Li \& Fernie, 2010). 
The length of the crossing will also have an impact: the longer the crossing, the less likely the violations (Cambon de Lavalette et al., 2009; Cinnamon et al., 2011). Land use and travel generators are also likely to have a correlation with the proportion of violations. At certain travel generators, violations have proven to be more common (Cinnamon et al., 2011; Zhuang\& Wu, 2011). Pedestrians crossing illegally will wait for an acceptable gap between oncoming traffic to safely cross the street. When conflicting vehicle flow increases, theses gaps become smaller and rarer, making it more difficult for pedestrians to cross the street (Wang et al.,2011; Yagil,2000; Yang et al., 2005).

Other factors, such as the day of the week, the period of the year or the time of day also impact the proportion of violations, as they are often linked to trip purpose and therefore change pedestrian behavior (Guo\&Wu, 2011; Wang et al., 2011). Weather will affect the level of comfort of a pedestrian and therefore have an impact on their crossing behavior (Li \& Fernie,2010; Yang et al., 2005). A study conducted in Toronto concluded that in winter conditions, people are less likely to wait at an intersection due to the cold. Other weather conditions, such as heavy rain, are also likely to shorten pedestrians' patience (Li \& Fernie, 2010).

The cultural context is also very important. Norms of conduct differ from one place to another, making it difficult to compare pedestrians from different cultural or social contexts (Rosenbloom,2009). Furthermore, most studies are done at a punctual place or in a specific area or city, making it difficult to generalize to other urban areas. Regardless, while results such as the proportion of violations or the average time a pedestrian will wait can rarely be directly applied across locations, factors associated with crossing behavior seem to be constant from one region to another.

Researchers have also explored how personal attitudes can be linked to violations, including violations of the rules of the road. The theory of planned behavior (TPB) is one of the most widely used social psychological models of health and safety related behavior (Ajzen, 1991): one's decision to engage in a particular behavior depends on one's attitude towards that behavior, subjective norms, and perceived behavioral control. The TPB has been applied to understand pedestrians' road crossing decisions, in particular through questionnaires. Evans and Norman (1998) identified all three components of the TPB as significant predictors of road crossing intentions in three scenarios, with perceived behavioral control being the strongest predictor. Moyano Diaz (2002) examined TPB in the task of crossing at mid-block: reported violations, errors and lapses appear causally related to the intention to violate traffic regulations. Young people have a more positive attitude towards committing violations, perceive subjective norms as less inhibitory while they have a lower perceived behavioral control. Zhou et al. (2009) also show that the tendency towards social conformity is associated with increased likelihood in crossing the road when other pedestrians were crossing. The variables of TPB, as well as perceived risk, were also significant predictors.

Despite this growing literature on pedestrian crossing behavior, in particular violations, few studies have looked at the link between violations and pedestrian waiting time, in particular in Chinese cities. To our knowledge, only one previous study (Van Houten et al., 2007) has looked at this issue, but their study examined two mid-block crosswalks using precontrolled times. Also, several other factors have been associated with the proportion of violations. However, to the extent of our knowledge, there have been very few studies that examine the association of pedestrian signal presence, time of arrival or maximum waiting time with the propensity to commit a violation. Studies on waiting time has not been discussed in detail, such as the time which they arrived at the beginning of the red and must wait the whole red phase or do they commit violations whatever their time of arrival. 
Moreover, violations are usually not differentiated with other dangerous crossings. Violations can be separated in different categories with different levels of risk. This is not to mention legal dangerous crossings that expose pedestrians to vehicles as much as certain types of violations.

\section{DEFINITIONS AND METHODOLOGY}

\subsection{The Definitions of Pedestrians' Violation}

The term 'violation' in the present research is used to denote any pedestrian crossings which are initiated during a yellow light, red light, steady hand, or flashing hand. The reader should note that countdown displays are excluded.

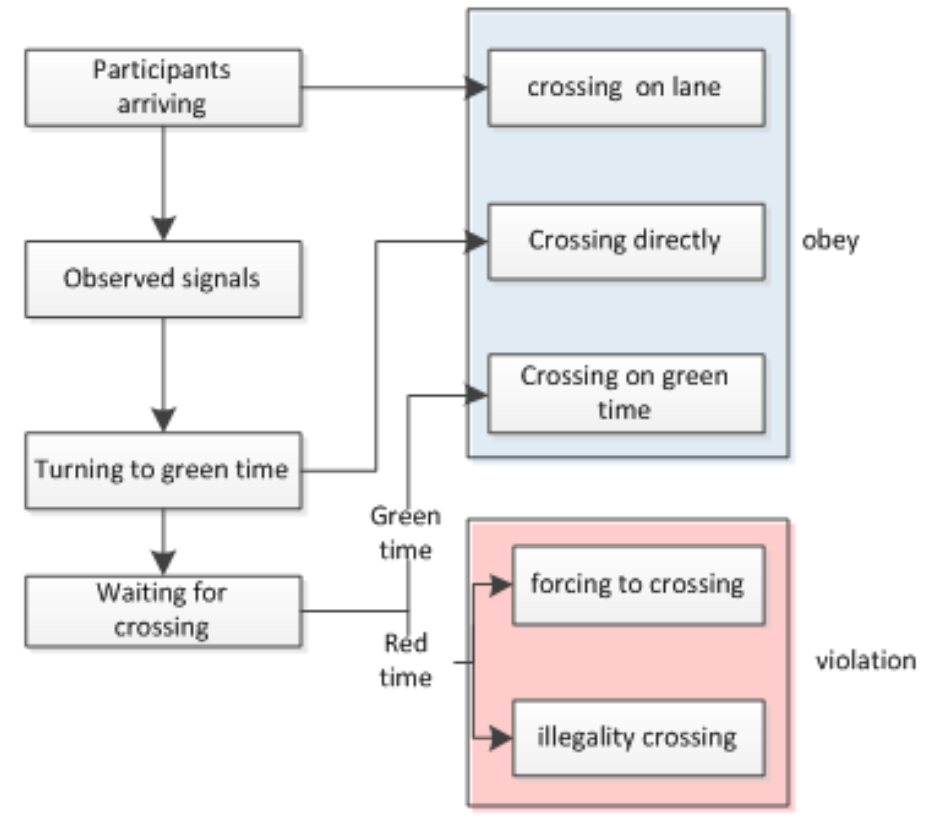

Figure.1 The process of pedestrians' violation

Hazardous crossings constitute crossings which are initiated during a red phase, whether partially or wholly. These are defined as hazardous owing to the way in which pedestrians engaging in these crossings are subjected to 'conflicting traffic', this term being used to denote a stream of vehicles which has the right of way during a red phase. It should be noted that conflicts also occur between pedestrians and turning vehicles in the course of the pedestrians' green phase, but this does not constitute a hazard because the vehicles anticipate crossing pedestrians. It is additionally noteworthy that patients have a 'continuous legal right of way' at intersections, which indicates that where a crossing is initiated during a green phase but completed during a red phase, this is not constitutive of a violation.

\subsection{The Factor of Crossing Violations}

The authors divide crossing behaviours into regular crossings, hazardous crossings, and crossing violations to facilitate a systematic investigation of pedestrian crossing behaviours at signalised intersections. Hazardous crossings and crossing violations are not mutually exclusive, thereby meaning that three sub-groups can be created as follows: 


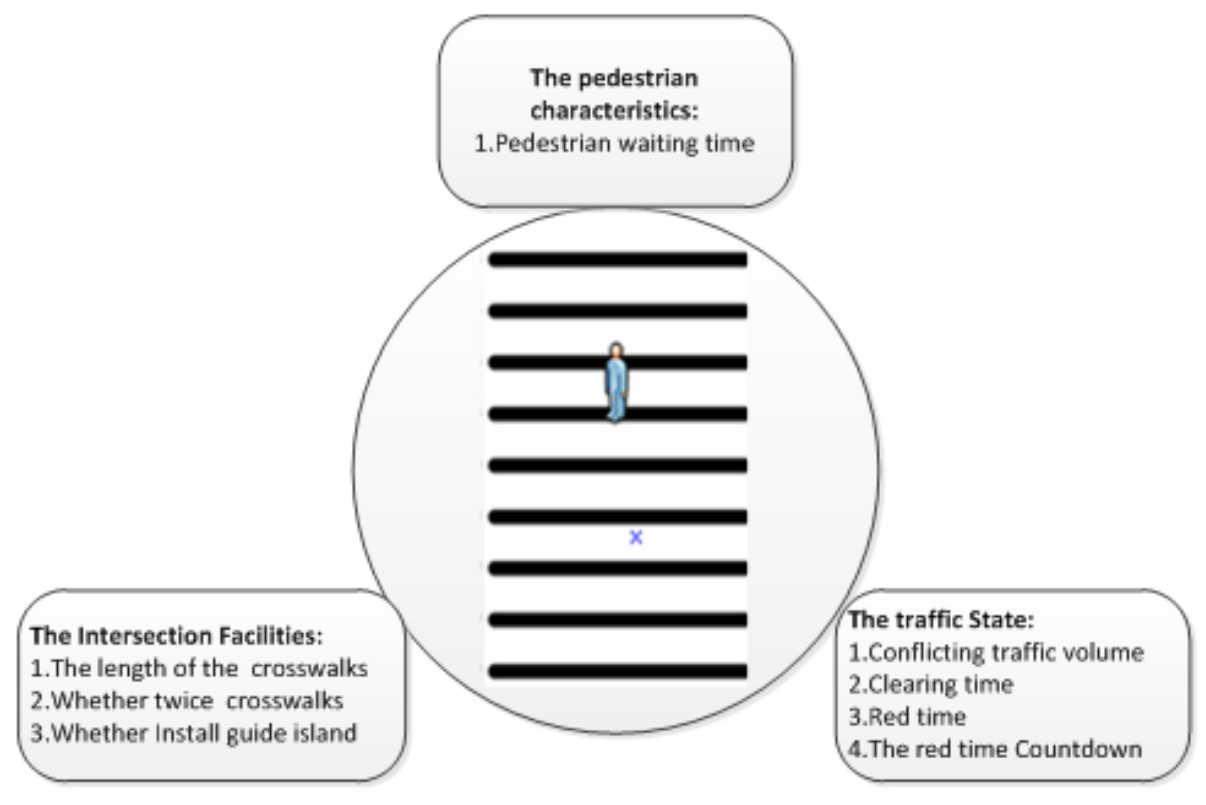

Figure .2 Field of Investigation

(a) Pedestrian Characteristics: Where crossings are initiated at any point of the red phase, a hazardous violation is considered as having taken place. There are two variants of this illegal crossing: namely, one which is initiated at the 'anticipation phase' (i.e. initiated overly early) or one which is initiated during the red phase.

(b) Intersection Facilities: One category of crossing violations is not hazardous because pedestrians are not subjected to conflicting traffic. Crossings of this type are initiated when the crossing takes place overly late (i.e. on the yellow light, flashing/full hand where a countdown display is not present) but completion takes place prior to conflicting traffic receiving the green light.

(c) Traffic State: A crossing is considered legal in cases where pedestrians initiate the crossing during the green phase or during the walking man signal, irrespective of whether completion occurs during the red phase. Despite the pedestrians' continuous legal right of way in this circumstance, a hazard can be presented by conflicting traffic.

\subsection{Site Selection}

As displayed in Figure 3, 13 intersection crossings have been chosen by the authors in Suzhou, China. These are representative of the characteristic traffic and roadway environments found in Chinese urban spaces. The intersections are situated in diverse contexts, such as malls, housing areas, and office blocks, and each intersection has a distinct configuration along with different traffic conditions. The defining features of each intersection is listed in Table 1.

As noted, each intersection is in Suzhou, China, and each of the identified 13 is situated on a prominent arterial: specifically, 12 are situated on Haiyubei Road and 1 is situated on Huanghe Road. The underlying rationale for this decision was that each intersection should have a comparable conflicting vehicle flow, geometry, and context. The choice of day was appropriate as these days of the week generally experience comparable traffic conditions. In this case, the specified window was June 21 to July 5, 2015. A series of key points to consider determined the time of day that data collection would take place, which was set as 09:30 to 11:30, the points being linked to the typical features of peak and non-peak times. It should also be noted that 
intersection choice was informed by a consideration of maximum waiting times; specifically, each of the 13 offers a broad range of maximum waiting times.

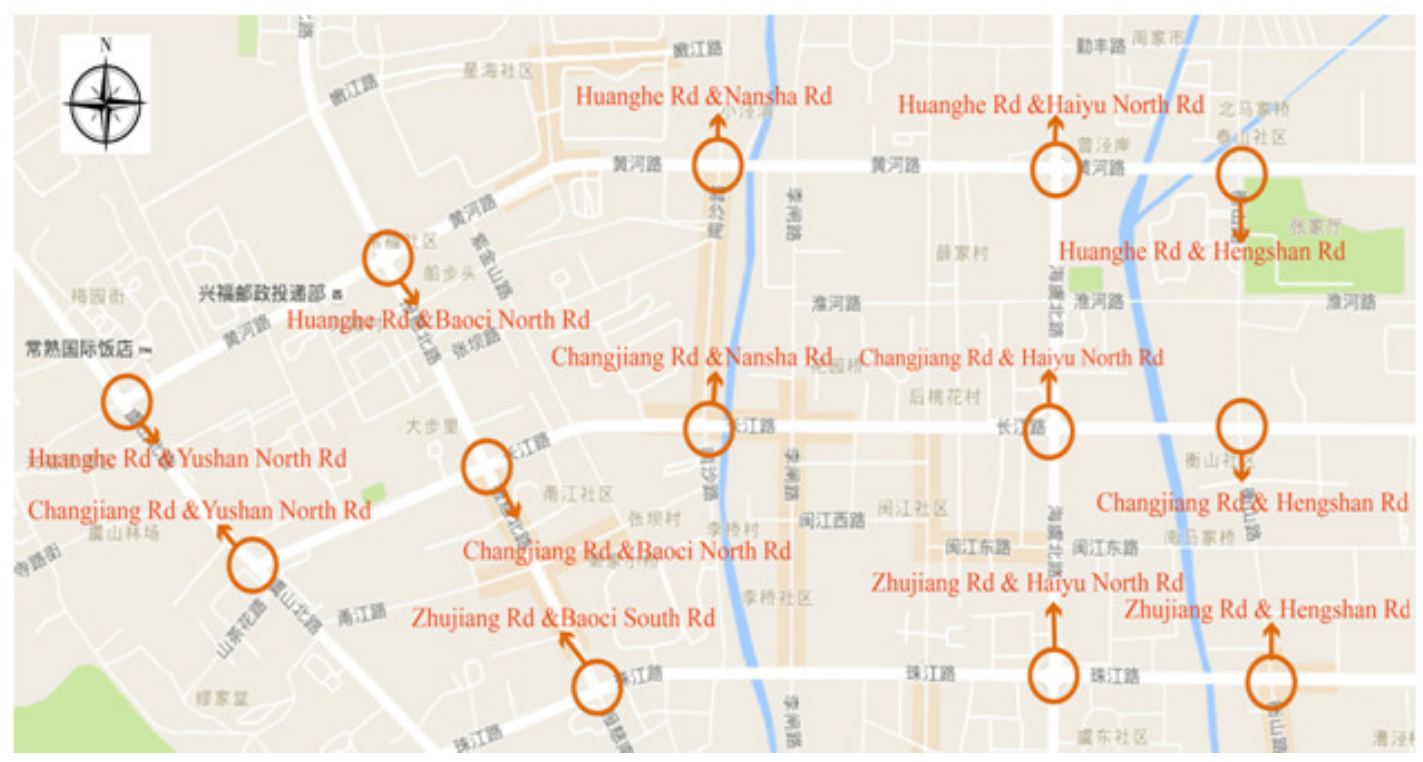

Figure. 3 Location of Selected Intersections.

Each category of intersection incorporates a minimum of one intersection with a pedestrian signal, the sole deviation being the short category; this stems from the fact that no comparable intersection exists in the target site which has a short and a pedestrian signal. Each with a varying kind of pedestrian signal: standard, with a call button, and with a countdown display. Figure 4 and Table 2 summarise each intersection's data, including location and features.

Counting took place in manual fashion using teams comprised of two individuals: one person was responsible for counting pedestrians, the other for counting violations and hazardous crossings. Hence, violations and hazardous crossings were incorporated into each dataset. The list below presents the categories of the gathered data:

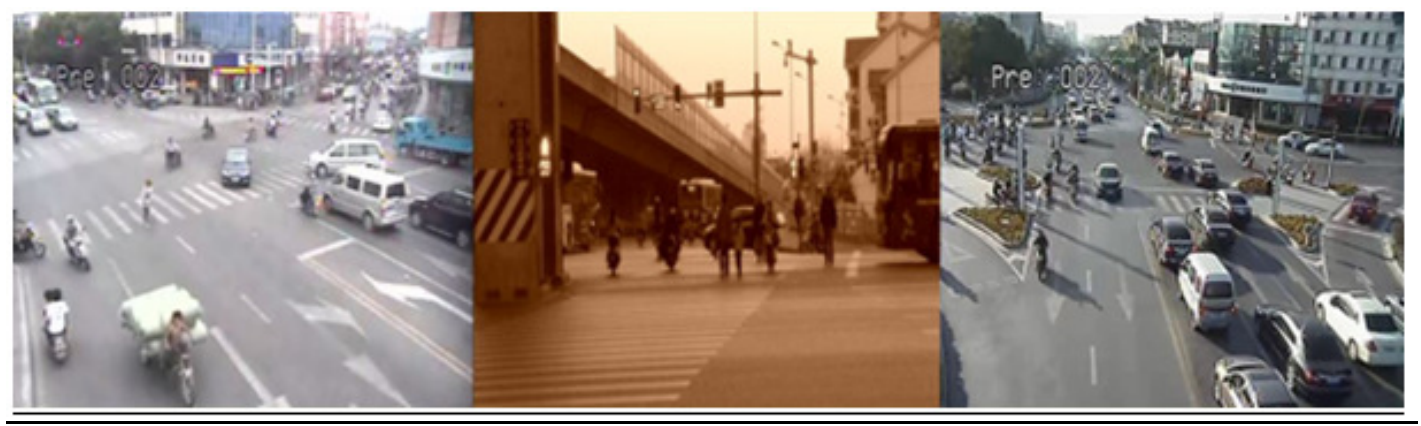

(a) once crosswalk

(b) twice crosswalk

(c)Install refuge islands

Figure. 4 Three Kinds of Crosswalks.

- time of arrival (time at which a pedestrian arrives to the crossing),

-age group,

- sex,

Age group was estimated in four age groups: 
Civil Engineering and Urban Planning: An International Journal (CiVEJ ) Vol.4, No.2, June 2017

-teenager(age 0-17),

•young adult (age 18-35),

-adult (age 36-59),

-senior (age 60+).

Table 1. Characteristics of Selected Intersections

\begin{tabular}{llllll}
\hline Num & Intersection & $\begin{array}{l}\text { Red } \\
\text { time } \\
\text { tr(s) }\end{array}$ & $\begin{array}{l}\text { Clearing } \\
\text { time tcl(S) }\end{array}$ & Investigation Period & $\begin{array}{l}\text { Pedestrians' } \\
\text { Violation } \\
\text { Rate(\%) }\end{array}$ \\
\hline 1 & Huanghe Rd/Xinshijie Rd & 90 & 4 & $7: 00 \mathrm{am}-9: 30 \mathrm{am}$ & 3.2 \\
2 & Beimen Rd/Yinxian Rd & 80 & 4 & $11: 30 \mathrm{am}-12: 30 \mathrm{am}$ & 13.1 \\
3 & Huanghe Rd/Cibei Rd & 120 & 4 & $5: 30 \mathrm{pm}-7: 00 \mathrm{pm}$ & 35.4 \\
4 & Yushannorth Rd/Huanghe Rd & 73 & 4 & $7: 00 \mathrm{am}-9: 30 \mathrm{am}$ & 22.1 \\
5 & Huanghe Rd/Haiyu north Rd & 80 & 6 & $11: 30 \mathrm{am}-12: 30 \mathrm{am}$ & 25 \\
6 & Zhaoshang East Rd/Shanghai Rd & 80 & 4 & $5: 30 \mathrm{pm}-7: 00 \mathrm{pm}$ & 4.2 \\
7 & Yushannorth Rd/Zhujiang Rd & 85 & 5 & $7: 00 \mathrm{am}-9: 30 \mathrm{am}$ & 6.3 \\
8 & Yushan north Rd/Changjiang Rd & 150 & 15 & $11: 30 \mathrm{am}-12: 30 \mathrm{am}$ & 26.9 \\
9 & Yushannorth Rd/Fenglin Rd & 100 & 17 & $5: 30 \mathrm{pm}-7: 00 \mathrm{pm}$ & 8.6 \\
10 & Ximen Rd/Shuyuan Rd & 65 & 18 & $7: 00 \mathrm{am}-9: 30 \mathrm{am}$ & 31.9 \\
11 & Qinhu Rd/Huaxi Rd & 60 & 16 & $11: 30 \mathrm{am}-12: 30 \mathrm{am}$ & 6.3 \\
12 & Haiyusouth Rd/Xinyan Rd & 70 & 15 & $5: 30 \mathrm{pm}-7: 00 \mathrm{pm}$ & 22.6 \\
13 & Huancheng Rd/Fuan Rd & 55 & 17 & $7: 00 \mathrm{am}-9: 30 \mathrm{am}$ & 34.5 \\
\hline
\end{tabular}

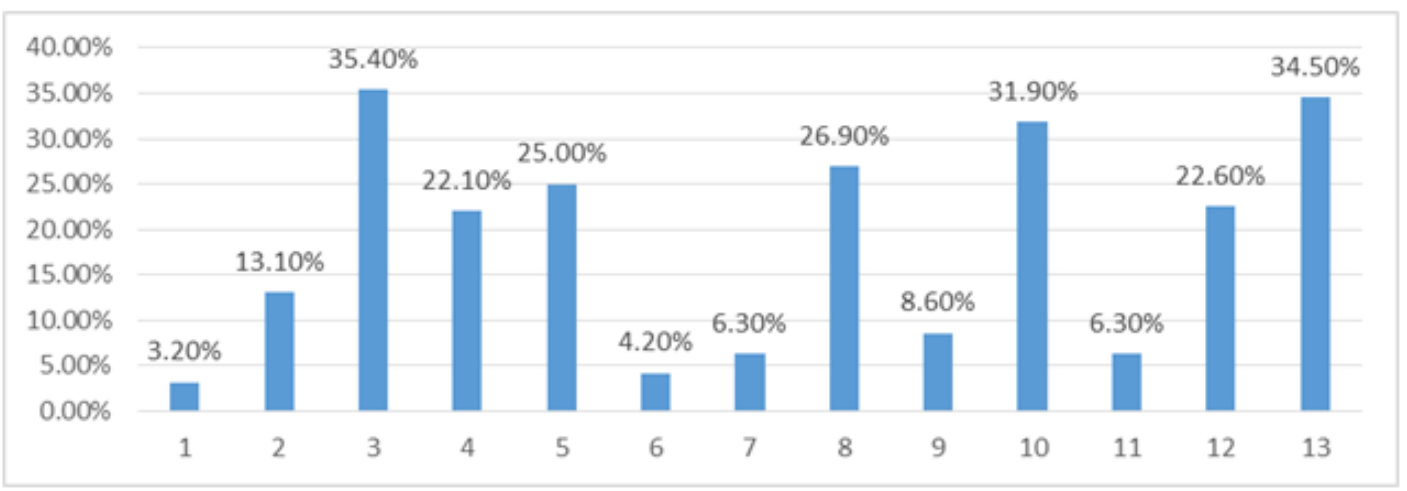

Figure 5. Pedestrians' Violation Rates in Selected Intersections

\subsection{Waiting Time Survey and Statistics}

During pedestrian tracking, crossing behaviour was recorded, and Figure 6 provides a schematic illustration of the way in which waiting time to crossing violation was calculated. A hazardous crossing violation is considered to have taken place when a pedestrian initiates a crossing during conflicting traffic's green or yellow phase, and this risk is referred to as an 'event'. Prior to initiating the crossing, the pedestrian waits for a period of time, and this is denoted as the 'waiting time'. To be specific, it is equivalent to the time elapsed between the pedestrian's arrival at the intersection and their initiation of the crossing.

To investigate the event of time to crossing violation, the researchers have solely taken into account pedestrians who arrive in the course of the hazardous crossing period. In this way, 
Civil Engineering and Urban Planning: An International Journal (CiVEJ ) Vol.4, No.2, June 2017

pedestrians $\mathrm{C} 1$ and $\mathrm{C} 2$ are not incorporated into the consideration. The minimum waiting time to crossing is considered in conjunction with the 'censoring time', which constitutes the point of time after which the crossing's hazardous nature has been eliminated and before the vehicle's red phase. Consequently, pedestrians X1, X2, V1, and V2 are incorporated into the analysis because they arrive in the course of the hazardous period. A distinction should be noted regarding X1 and $\mathrm{X} 2$ in that their decision to wait until the safe crossing period to initiate their crossing means that it is regarded as safe when the hazardous phase is completed.

One of the key findings generated from this observation is that the number of pedestrians travelling in conjunction is associated with the frequency of violation. A manifest representation of this is reflected in Figure 3 such that the number of violations rises as the size of the group rises. It is notable that a comparable trend was found for several pedestrians who initiated their crossing at the corner of the intersection; it is important to note that this has not been listed as a key finding because much of the data remained uncollected, primarily owing to the complexity of gathering accurate data regarding this variable.

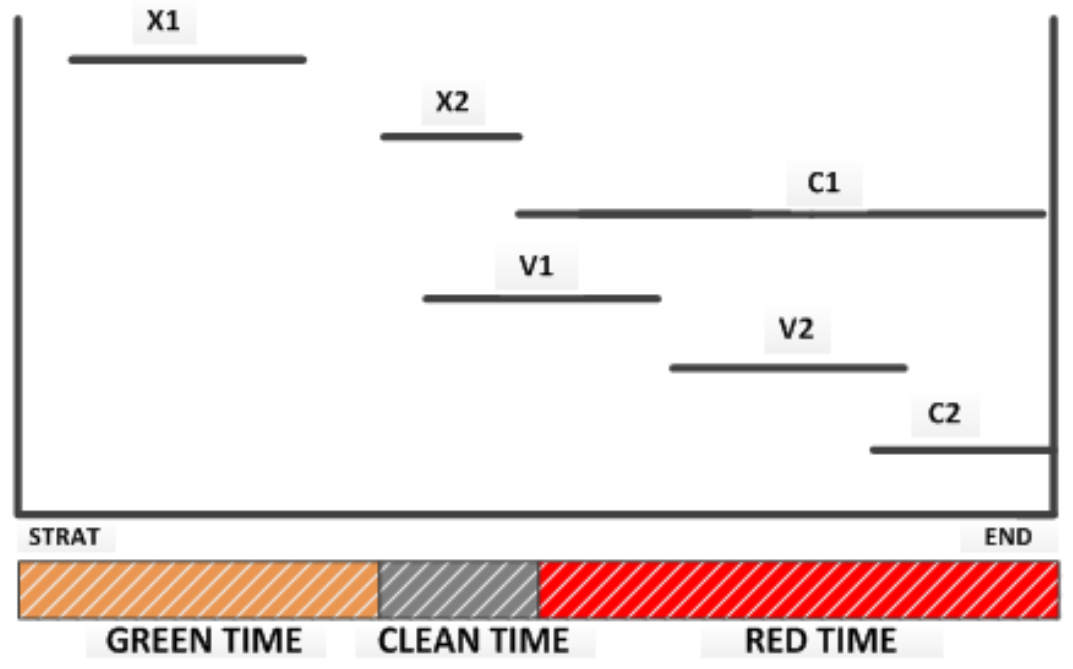

Figure 6. Schematic Diagram of Time to Violation Crossing

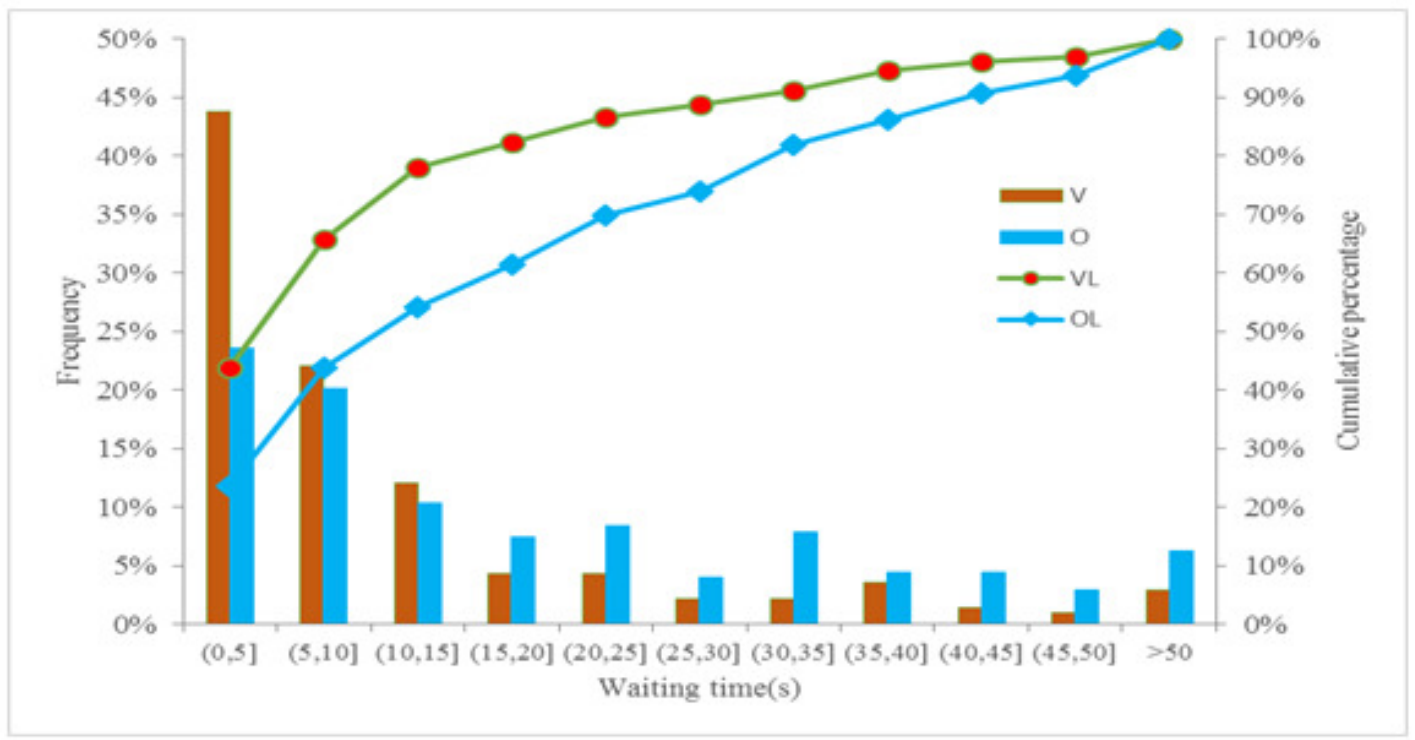

Figure 7. The Distribution of Waiting Time in Intersection Survey 


\section{METHODOLOGY}

\subsection{Model Structure}

Pedestrian behavioural modelling at signalised intersections routinely draws on discrete choice models. The goal of the present research is to model the gap acceptance decisions engaged in by pedestrians based on a range of conditions, including environmental, geometric, and traffic. In view of this, binary logit models have been employed to facilitate effective modelling, and presents a comprehensive overview of these models in combination with other discrete choice models. With the employment of a binary logit model, the likelihood of the acceptance of a given $\mathrm{i}$ by pedestrian $\mathrm{k}$ is expressed in Eq. (1-2).

$$
\begin{aligned}
& P_{n}(1)=\frac{e^{V_{1 n}}}{e^{V_{1 n}}+e^{V_{2 n}}} \\
& U_{i n}=V_{i n}+\varepsilon_{i n}
\end{aligned}
$$

$\mathrm{U}_{\mathrm{i}}$ represents the utility of pedestrian $\mathrm{i}$, expressed as (Eq. (3)):

$$
V_{i n}\left(x_{i n}, \beta\right)=\beta_{1} x_{i n 1}+\beta_{2} x_{i n 2}+\beta_{3} x_{i n 3}+\cdots+\beta_{K} x_{i n K}
$$

where $\mathrm{X} 1, \mathrm{X} 2, \ldots, \mathrm{Xn}$ constitute the pedestrian's decision-influencing variables and $b_{1}, b_{2}, \ldots, b_{n}$ represent associated coefficients.

For the purpose of facilitating the development of binary logit models, the authors drew on NLOGIT, an econometric and statistical instrument.

\subsection{Model estimation}

The estimation of the model presented in Eq. (4) takes place by utilising full information maximum. The log-likelihood function that is purposed to be maximised is as follows:

$$
L=\sum_{n=1}^{N} \sum_{i=1}^{I} y_{n i} \ln P_{n i}
$$

where I represent the number of violating pedestrians; $\mathrm{N}$ denotes the number of pedestrians; $\mathrm{P}_{\mathrm{ni}}$ is given by (4) and $\mathrm{y}_{\mathrm{ni}}$ is defined as 1 if the nth pedestrian violates; 0 otherwise.

\subsection{Model Development}

In terms of the factors linked to traffic control, it is possible to discern a clear correlation between every form of pedestrian signal and the considerable reduction of the likelihood of a hazardous crossing and violation. This finding is also reported in (23). The present study's findings further replicate the findings reported in (26), according to which the presence of a countdown signal is correlated with the likelihood of violation in a negative way. The likelihood of violation or hazardous crossing at intersections with a countdown signal is approximately $15 \%$ less than those lacking a signal of this kind. Furthermore, the data suggests that MWT influences violations; to 
Civil Engineering and Urban Planning: An International Journal (CiVEJ ) Vol.4, No.2, June 2017

be specific, a rise in $10 \%$ for MWT in this dataset is correlated with corresponding rises of $7.9 \%$ regarding the likelihood of violation and $2.1 \%$ regarding the likelihood of hazardous violation.

The data indicates that group size and pedestrian flow are negatively correlated with the likelihood of violations; a reduction in the former or the latter variable by $10 \%$ is linked to a corresponding reduction in the probability of violations and hazardous violations by up to $0.9 \%$ and $0.6 \%$, respectively. Furthermore, as previously noted, males are more likely to engage in violations when crossing than their female counterparts. Additionally, age influences the probability associated with violation, where young adults are associated with the greatest likelihood of violation or hazardous violation, thereby corroborating the extant literature regarding higher prevalence among young adults (28), males (20), and smaller groups (22).

Table 2. Parametric Binary Logit Regression Results

\begin{tabular}{|c|c|c|c|c|c|}
\hline & Coef. & S.E. & Wals & $\mathrm{P}>\mathrm{z}$ & $\operatorname{Exp}(B)$ \\
\hline constant & 0.35 & 0.44 & 0.61 & 0.03 & 1.42 \\
\hline gender & 0.22 & 0.13 & 2.91 & 0.05 & 1.24 \\
\hline \multicolumn{6}{|l|}{ Age } \\
\hline teenager & -0.065 & 0.08 & 0.38 & 0.54 & 0.95 \\
\hline young adult & -0.111 & 0.07 & 0.24 & 0.11 & 1.11 \\
\hline adult & -0.169 & 0.08 & 0.18 & 0.18 & 1.39 \\
\hline senior & -0.194 & 0.05 & 0.20 & $0.00^{*}$ & 1.21 \\
\hline waiting time & 0.33 & $0.00 *$ & 4.87 & $0.00^{*}$ & 1.03 \\
\hline lane & -0.13 & 0.06 & 4.33 & 0.02 & 0.88 \\
\hline red time & $0.00^{*}$ & 0.01 & 1.15 & 0.04 & 0.99 \\
\hline \multicolumn{6}{|l|}{ Crossing type } \\
\hline once crosswalk & 0.48 & 0.09 & 3.95 & 0.05 & 1.20 \\
\hline twice crosswalks & 0.35 & 0.05 & 2.94 & 0.03 & 1.09 \\
\hline install refuge islands & 0.33 & 0.07 & 2.84 & 0.01 & 1.08 \\
\hline \multicolumn{6}{|l|}{$\mathrm{N}=1095$} \\
\hline \multicolumn{6}{|l|}{ Log likelihood: } \\
\hline \multicolumn{6}{|l|}{$\mathrm{L}(0)=723.12$} \\
\hline \multicolumn{6}{|l|}{$L(\beta)=511.97$} \\
\hline Pseudo R2 0.29 & & & & & \\
\hline
\end{tabular}

Note: * significance at the $5 \%$ level

S.E. stands for Standard Error of Mean

\section{CONCLUSION}

The aim of this research has been to assess the effect that waiting time - determined by phasing length and arrival time - arrival time, and proximity to a pedestrian signal have on the degree to which pedestrians commit violations during intersection crossings. Increases in the frequency of violations are correlated with a range of variables while the converse is also true: specifically, males, young adults, and higher intersection times are associated with higher frequencies; contrastingly, pedestrian signal presence and group size are associated with reduced frequencies. In view of these findings, it is possible to conclude that crosswalk type and waiting time are critical matters to consider when formulating viable ways to resolve the issue of pedestrian violations. 
Consequently, when programming cycle length and viable green/red periods for the respective phases, a close focus should be directed towards pedestrian waiting times. Certain variables have been shown to influence the category of violation committed, thereby meaning that violations and hazardous violations can be mitigated against by aligning the waiting time to the average time taken to complete a crossing.

Further studies could attempt to replicate the results reported by these authors by conducting larger-scale examinations (for example, in terms of intersection number) in a range of cities in China. The limitations associated with the manual mode of data collection - linked to transcription errors, the inability to accurately log every pedestrian, and misevaluating pedestrian age - could also be improved. Furthermore, the generalisability of the study is impaired by the exclusive focus on intersections in the city centre of Suzhou, China. An expanded scope constitutes a promising area of future investigation.

\section{ACKNOWLEDGEMENTS}

This study is supported by the National Natural Science Foundation of China (No.61374195).

\section{REFERENCES}

[1] Ajzen, I. The theory of planned behavior. Organizational Behavior and Human Decision Processes, (1991).50, 179-211.

[2] Elvik, R. The non-linearity of risk and the promotion of environmentally sustainable transport. Accident Analysis and Prevention, (2009).41(4), 849-855.

[3] Harwood, D., Bauer, K., Richard, K., Gilmore, D., Graham, J., \& Potts, I. Pedestrian Safety Prediction Methodology. NCHRP Project 17-26. (2008).

[4] Miranda-Moreno, L. F., Morency, P.,\& El-Geneidy,A.M.The link between built environment, pedestrian activity and pedestrian-vehicle collision occurrence at signalized intersections. Accident Analysis and Prevention, 43(5), 1624-1634.(2011).

[5] Guo, H., Gao, Z., Yang, X., \& Jiang, X. Modeling pedestrian violation behavior at signalized crosswalks in China: A hazards-based duration approach.Traffic Injury Prevention (2011)., 96-103.

[6] Li, Y., \& Fernie, G. Pedestrian behavior and safety on a two-stage crossing with a center refuge island and the effect of winter weather on pedestrian compliance rate. Elsevier Accident Analysis and Prevention, 1156-1163.(2010).

[7] Ren, G., Zhou, Z., Wang, W., Zhang, Y., \& Wang, W. Crossing behaviors of pedestrians at signalized intersections. Transportation Research Record: Journal of the Transportation Research Board, 2264, 65-73. (2011).

[8] Rosenbloom, T. Crossing at a red light: Behaviour of individuals and groups. Elsevier Transportation Research Part F, 389-394. (2009).

[9] Van Houten, R., Ellis, R. \& Kim, J. L. The effects of varying minimum green on the percentage of pedestrians waiting to cross with the WALK signal at mid-block crosswalks. Transportation Research Record, No. 2002-10, 78-83). (2007).

[10] Bierlaire, M. BIOGEME: A free package for the estimation of discrete choice models. In Proceedings of the 3rd Swiss Transportation Research Conference, Ascona, Switzerland. (2003).

[11] Bierlaire, M. An introduction to BIOGEME version 1.6. biogeme.epfl.ch.(2008). 
Civil Engineering and Urban Planning: An International Journal (CiVEJ ) Vol.4, No.2, June 2017

[12] Cambon de Lavalette, B., Tijus, C., Poitrenaud, S., Leproux, C., Bergeron, J., \& Thouez, J.-P, (2009)Pedestrian crossing decision-making: A situational and behavioral approach. Elsevier Safety Science. 1248-1253.

[13] Cinnamon, J., Schuurman, N., \& Hameed, S. M. (2011). Pedestrian injury and human behaviour: Observing road-rule violations at high-incident intersections.

[14] Elvik, R. (2009)The non-linearity of risk and the promotion of environmentally sustainable transport. Accident Analysis and Prevention.41(4), 849-855.

[15] Evans, D., \& Norman, P. (1998). Understanding pedestrians' road crossing decisions: An application of the theory of planned behavior. Health Education Research,vol. 13. Oxford University Press (pp. 481-489). Oxford University Press.

[16] Government of Quebec Highway safety code. Consultéle (2011). on Publications du Québec.

[17] Guo, H., Gao, Z., Yang, X., \& Jiang, X. (2011). Modeling pedestrian violation behavior at signalized crosswalks in China: A hazards-based duration approach.Traffic Injury Prevention, 96-103.

[18] Harwood, D., Bauer, K., Richard, K., Gilmore, D., Graham, J., \& Potts, I. (2008). Pedestrian Safety Prediction Methodology. NCHRP Project 17-26.

[19] Ismail, K., Sayed, T., \& Saunier, N. (2010). Automated analysis of pedestrian-vehicle conflicts: Context for before-and-after studies. Transportation Research Record: Journal of the Transportation Research Board, 2198, 52-64.

[20] Jackson, S., Miranda-Moreno, L., St-Aubin, P. \& Saunier, N. (2013). A flexible, mobile video camera system and open source video analysis software for road safety and behavioural analysis. Transportation Research Board Annual Meeting Compendium of Papers.

[21] Li, Y., \& Fernie, G. (2010). Pedestrian behavior and safety on a two-stage crossing with a center refuge island and the effect of winter weather on pedestrian compliance rate. Elsevier Accident Analysis and Prevention, 1156-1163.

[22] Markowitz, F., Sciortino, S., Fleck, J. L., \& Yee, B. (2006). Pedestrian countdown signals: Experience with an extensive pilot installation. ITE Journal, 43-48.

[23] Miranda-Moreno, L. F., Morency, P.,\& El-Geneidy,A.M. (2011).The link between built environment, pedestrian activity and pedestrian-vehicle collision occurrence at signalized intersections. Accident Analysis and Prevention, 43(5), 1624-1634.

[24] Moyano Diaz, E. (2002).Theory of planned behavior and pedestrians' intentions to violate traffic regulations.Transportation Research Part F:Traffic Psychology and Behaviour, 5, 169-175.

[25] Ren, G., Zhou, Z., Wang, W., Zhang, Y., \& Wang, W. (2011). Crossing behaviors of pedestrians at signalized intersections. Transportation Research Record: Journal of the Transportation Research Board, 2264, 65-73.

[26] Rosenbloom, T. (2009).Crossing at a red light: Behaviour of individuals and groups. Elsevier Transportation Research Part F, 389-394.

[27] Saunier, N., \& Sayed, T. (2006). A feature-based tracking algorithm for vehicles in intersections. IEEE.

[28] Tiwari, G., Bangdiwala, S., Saraswat, A., \& Gauray, S. (2007). Survival analysis: Pedestrian risk exposure at signalized intersections. Elsevier TransportationResearch Part F, 77-89. 
Civil Engineering and Urban Planning: An International Journal (CiVEJ ) Vol.4, No.2, June 2017

[29] Van Houten, R., Ellis, R. \& Kim, J. L. (2007). The effects of varying minimum green on the percentage of pedestrians waiting to cross with the WALK signal at mid-block crosswalks. Transportation Research Record, No. 2002-10, 78-83).

[30] Wang, W., Guo, H., Gao, Z., \& Bubb, H. (2011). Individual differences of pedestrian behaviour in midblock crosswalk and intersection. International Journal of Crashworthiness, 1-9.

[31] Wanty, D., \& Wilkie, S. M. (2010) Trialling pedestrian countdown timers at traffic signals. Wellington: NZ Transport Agency Research Report 428.

[32] Zhuang, X., \& Wu, C. Pedestrians' crossing behaviors and safety at unmarked roadway in China. Elsevier Accident Analysis and Prevention, 1927-1936 (2011).

[33] Brilon, W., Koenig, R., \& Troutbeck, R. J. (1999). Useful estimation procedures for critical gaps. Transportation Research Part A: Policy and Practice, 33(3), 161-186.

[34] Ben-Akiva, M. E., \& Lerman, S. R. (1985). Discrete choice analysis: Theory and application to travel demand. MIT Press, 9.

\section{AUTHORS}

Jiping Xing is currentlypursuingaPh.D.in Transportation Engineering in SouthEast University,China. He received M.E. degree in Suzhou University of Science and Technology In 2015.His current research interests include pedestrian safety.

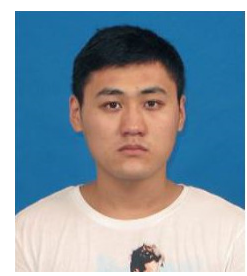

